\title{
X-raying the Universe
}

\author{
On the anniversary of ROSAT's launch, we reflect on the relevance and promise of X-ray observations in all fields of \\ astronomy. Properly laying out a plan for the future of the field will allow it to continue flourishing.
}

$\mathrm{O}$ n 1 June 1990, the ROSAT (Roentgen Satellite) facility launched from Cape Canaveral, effectively ushering in a new era for the field of X-ray astronomy by performing an all-sky survey that increased the number of known X-ray sources by orders of magnitude. Now, 30 years later, the landscape of X-ray astronomy has radically changed with a plethora of space telescopes, such as the Chandra X-ray Observatory, XMM-Newton, AstroSat, Hinode and AGILE, observing the sky at $\mathrm{X}$-ray energies. The latest addition to the roster of X-ray observatories has been the Russian-German Spectrum Roentgen Gamma (SRG) space mission, launched last year, which aims to improve on ROSAT's legacy with a new deep all-sky survey carried out by eROSITA, one of the two instruments aboard SRG.

A collaboration between the United States, the United Kingdom and Germany, ROSAT's all-sky survey in the X-rays (from around 0.1 to $2.4 \mathrm{keV}$ ) radically changed our view of the Universe with high angular resolution ( $\sim 5$ arcsecs) and a sensitivity that was orders of magnitude better than previous X-ray surveys of the sky. This survey resulted in a catalogue that contained more than 150,000 individual sources. Just to put things into perspective, the Orbiting Solar Observatory 7, operating between 1971 and 1974, was the first X-ray observatory to produce an all-sky catalogue at about 1 degree of angular resolution, which contained around 180 sources (including community favourites such as Cyg X-1 and Vela X-1). eROSITA is expected to detect up to four million sources!

Perhaps some of the most significant scientific contributions of X-ray astronomy have been in the study of compact objects and transient events associated with them. Observatories like NuSTAR and Suzaku have been key in the study of the astrophysics of black holes, both stellar and supermassive, as they can probe emission coming from the hot gas being accreted onto or orbiting these objects. The combination of sensitive imaging and spectroscopy enabled us to peer deep into the cores of active galactic nuclei and X-ray binaries to reveal general relativistic effects on the gas around these compact objects. The study of these small-scale gas flows in X-rays allowed the measurement of black hole spins (as reviewed by Chris Reynolds recently) and the discovery of ultrafast outflows close to the speed of light.

On larger scales, catalogues of sources like the ones produced by ROSAT and soon eROSITA enable us to identify accreting black holes in galaxies near and far, pushing all the way back to the cosmic dawn (the highest redshift X-ray quasar has been detected at $z=7.54$ ). This information can then be translated into an understanding of the demographics of black holes, how they formed, how they evolve and consequently how they may be affecting the formation and evolution of the galaxies that host them.

On the largest scales, X-ray observations allowed the discovery and study of hot gas within clusters of galaxies, the biggest gravitationally bound structures in the Universe. Apart from revealing that the hot gas component of clusters contains many times the visible baryonic mass within galaxy cluster members, X-ray observations also revealed the dynamic nature of clusters, including shocks and bubbles of gas, fuelled by energy input from accreting supermassive black holes. In addition, X-ray observations of clusters can be used as cosmological probes of the cosmic baryon content, as well as the cosmic expansion, dark matter and dark energy. Perhaps the most spectacular showcase of X-ray cluster science was performed by the ill-fated Hitomi satellite, which revealed in exquisite detail the complexity of the gas lifecycle within clusters. XRISM, Hitomi's successor to be launched in 2022, is expected to pick up the study of clusters in the X-rays where Hitomi left off.

Scientific applications of X-ray observations, however, do not stop there. The study of neutron stars, X-ray binaries and supernova remnants in the X-rays also reveals important information about the endpoints of stellar evolution, made all the more relevant by the recent efforts in understanding the genealogy and evolution history of the binary merger systems detected in gravitational waves by LIGO/ Virgo. The merger events themselves, as well as other energetic but transient phenomena, can be detected and uniquely characterized by X-ray observations with facilities such as the Neil Gehrels Swift Observatory, as well as NICER and MAXI aboard the ISS.

The importance of X-ray astronomy and specifically the study of hot gas in the Universe are reflected in this year's Kavli Prize for Astronomy. It was awarded to Andrew Fabian, for his contributions to $\mathrm{X}$-ray astronomy including his studies of flows of hot gas in clusters and black-hole spin measurements based on general relativistic effects on X-ray spectral emission lines. To commemorate this occasion, we have published a collection of papers authored by Fabian, his collaborators and others that cover both these scientific topics.

The outlook for X-ray astronomy is bright but not without its uncertainties. The next generation of flagship X-ray telescopes, including ESA's Athena and the proposed NASA's Lynx observatories, is planned to operate in the 2030s, at least ten years from today. Can the current generation of X-ray facilities survive until then? Both NASA's Chandra X-ray Observatory and ESA's XMM-Newton have now been operating for more than 20 years (far longer than their nominal lifetimes). With a rapidly ageing suite of instruments and mechanical parts, there is a real danger that a malfunction (such as the gyroscope glitch Chandra experienced in 2018) could leave the $\mathrm{X}$-ray astronomy community without a general-purpose observing facility for many years.

The diversity of X-ray astronomy was showcased in a recent meeting in Bologna (recorded in a Meeting Report by Martin Elvis).The treasure trove of information on the 'invisible' Universe afforded by the $\mathrm{X}$-ray vision of current facilities shows in a clear way not only the relevance of $\mathrm{X}$-ray astronomy to all established fields of astronomy, astrophysics and planetary science, but also its promise for future studies of the transient Universe, including gravitational waves and the emerging field of multi-messenger astronomy.

Published online: 12 June 2020

https://doi.org/10.1038/s41550-020-1137-9 